تقويم الأداء والارتباط والتحسين الوراثي المتوقع للحاصل ومكوناته في الباقلاء (Vicia faba L.

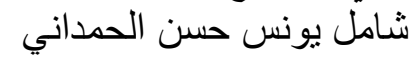

قسم البستنة وهندة الحدائق- كلية الزراعة و الغابات- جامعة الموصل- العراق

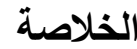

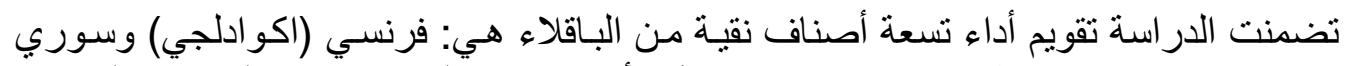

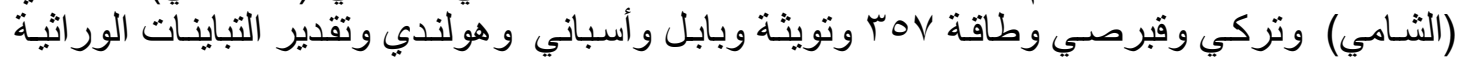

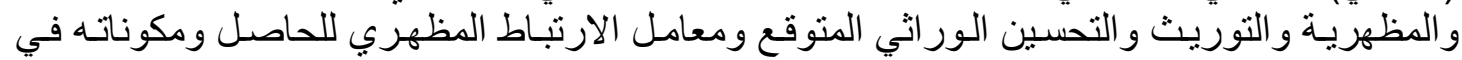

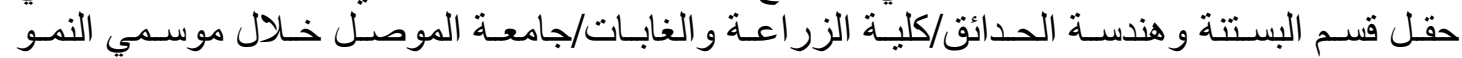

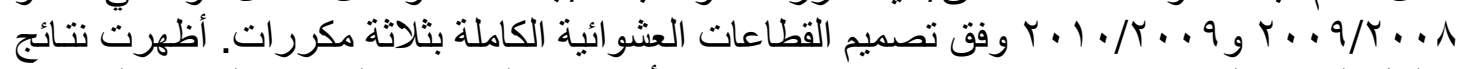

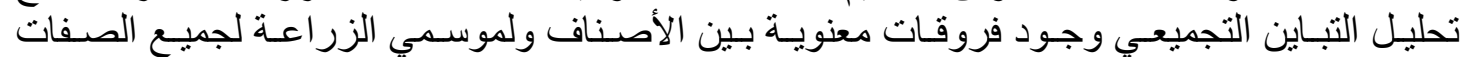

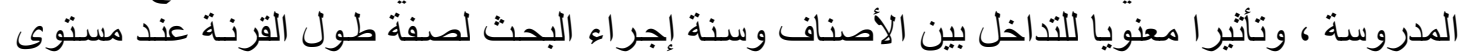

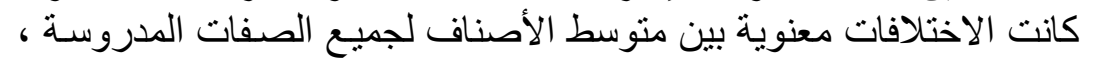

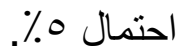

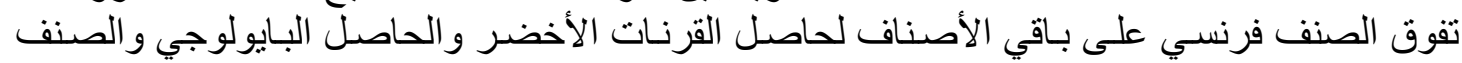

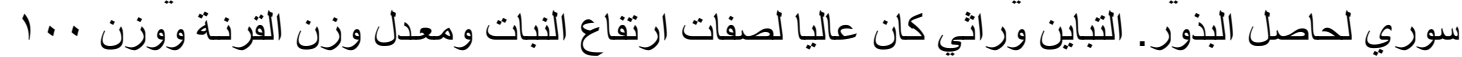

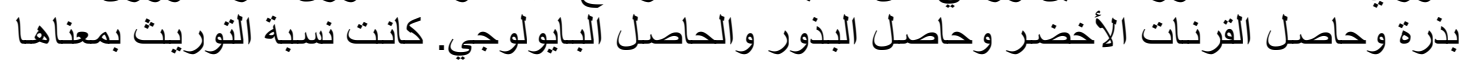

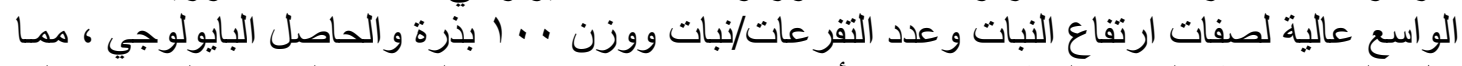

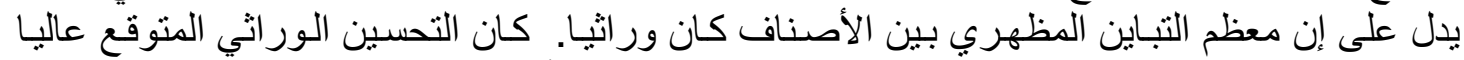

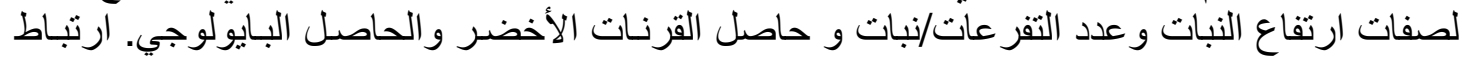

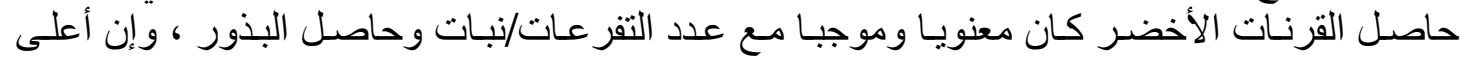

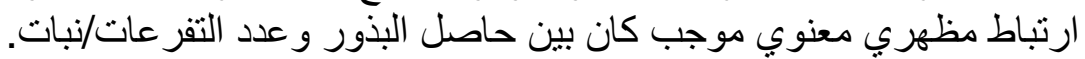

\section{المقدمة}

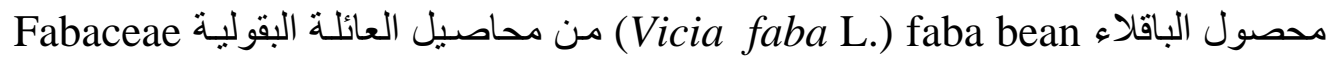

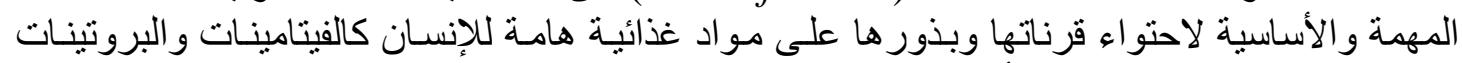

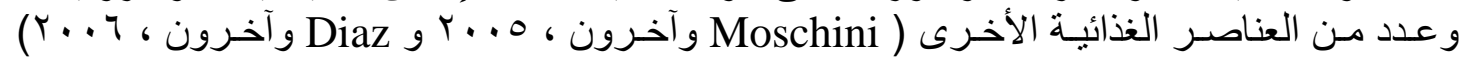

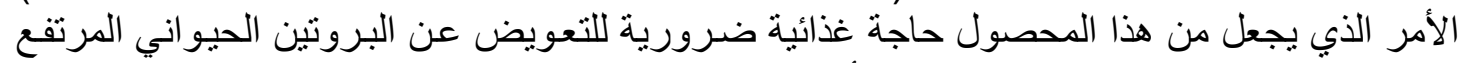

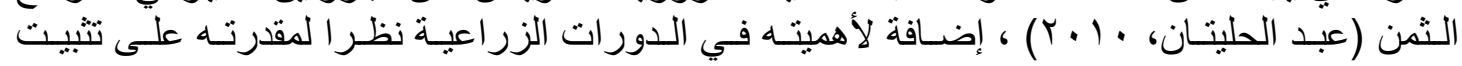

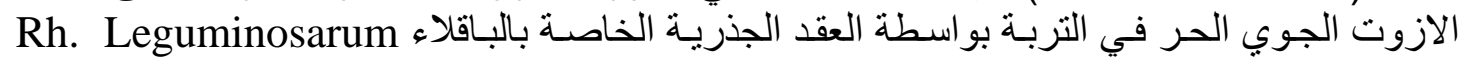
Kahalil)

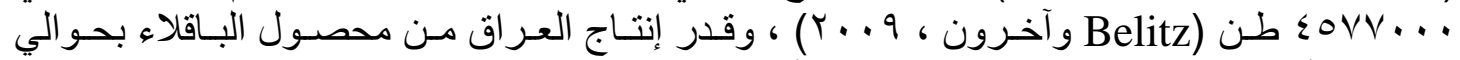

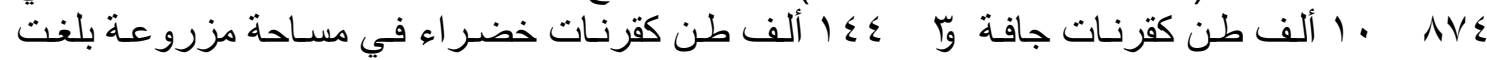

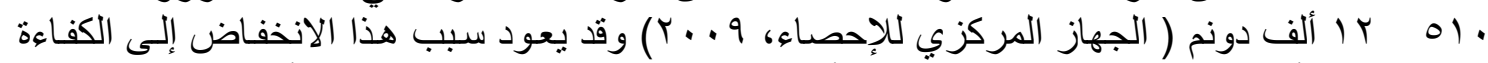

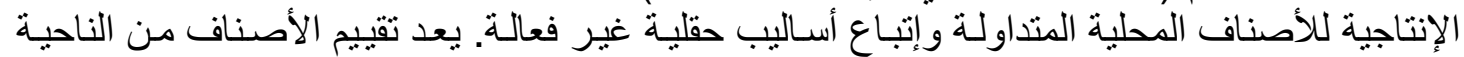

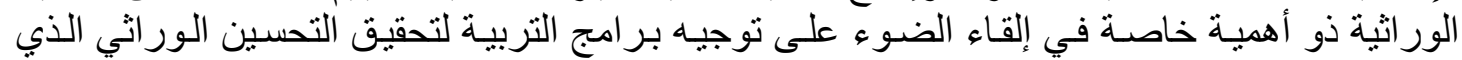

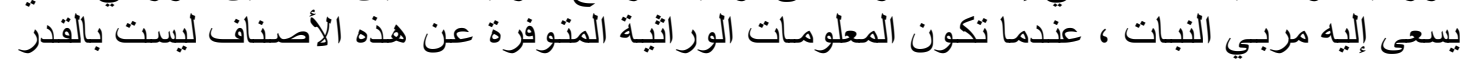

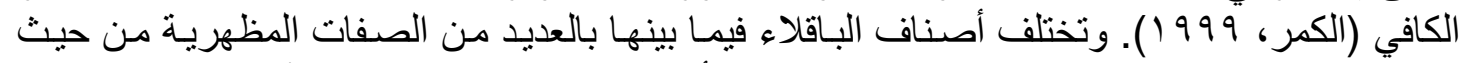

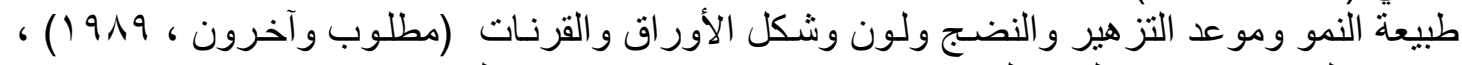

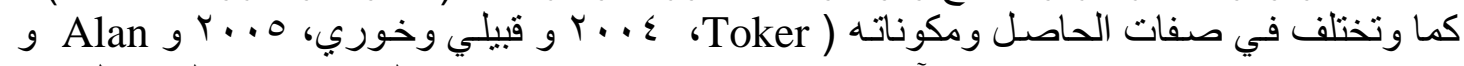

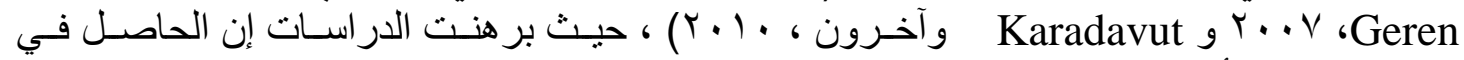

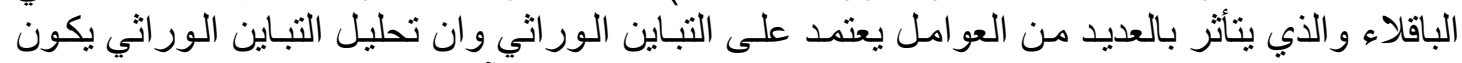

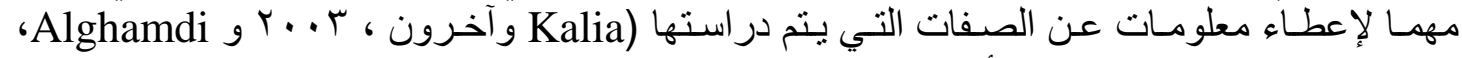

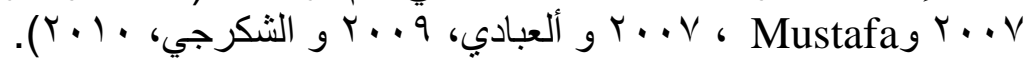

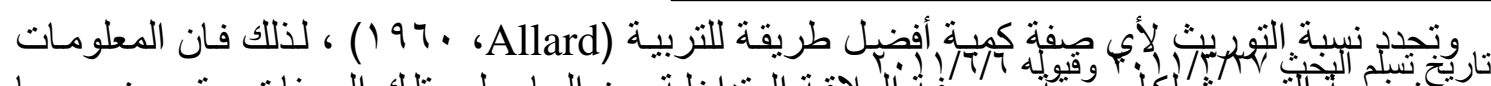

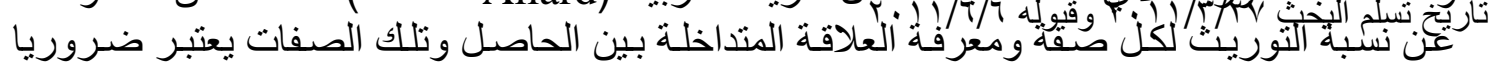




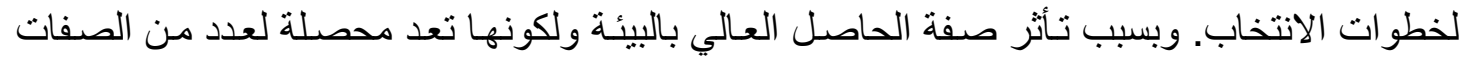

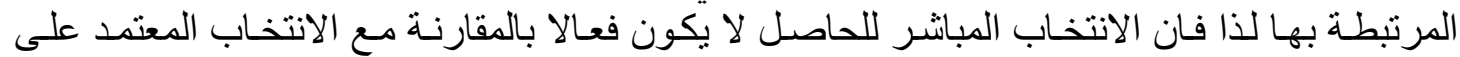

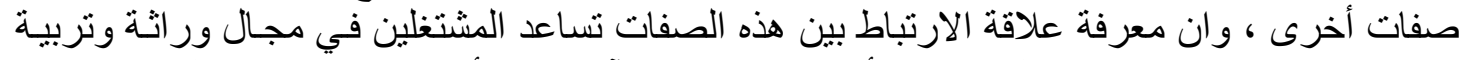

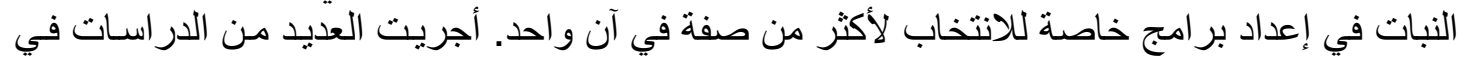

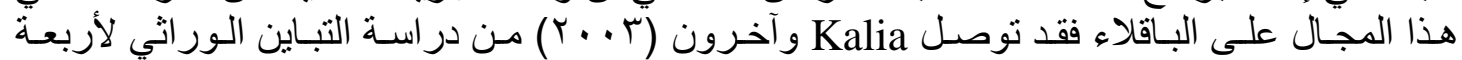

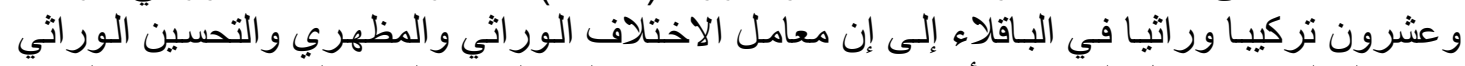

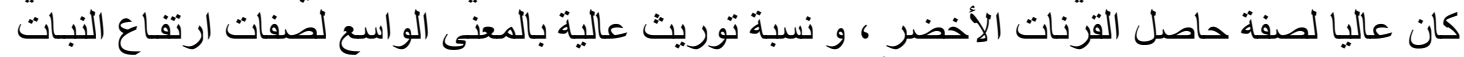

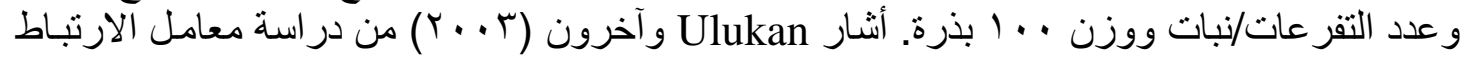

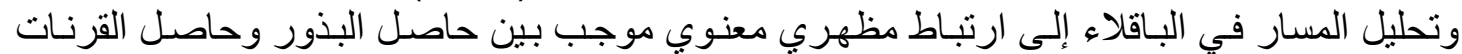

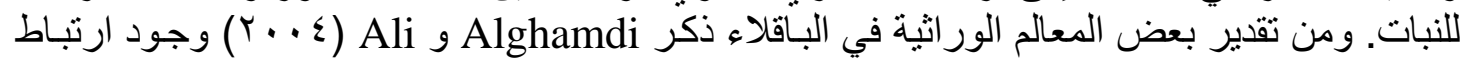

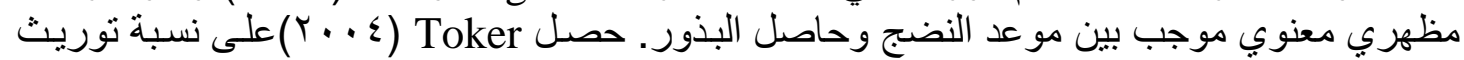

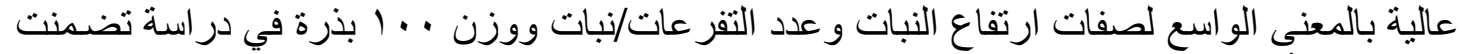

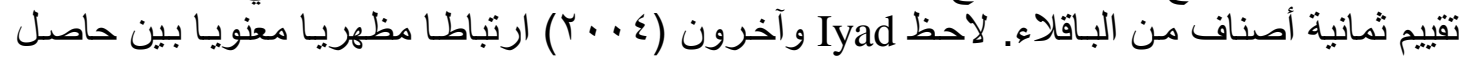

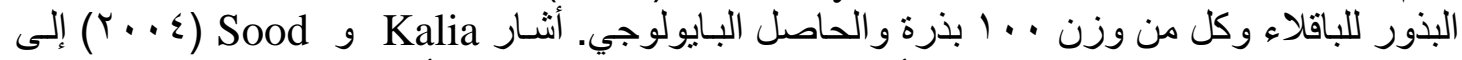

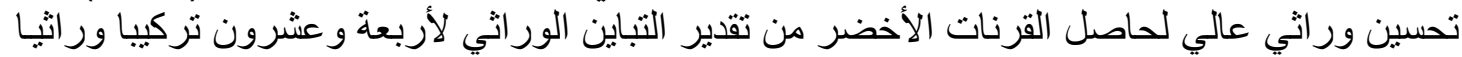

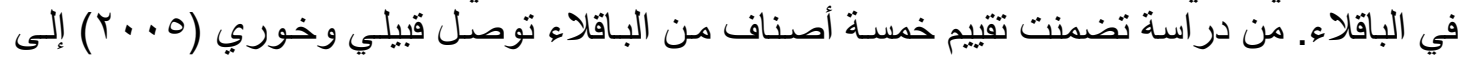

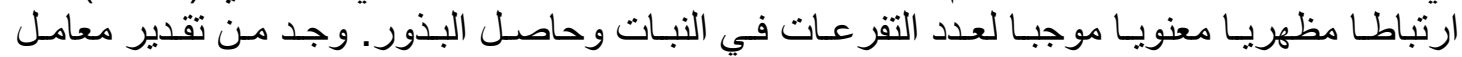

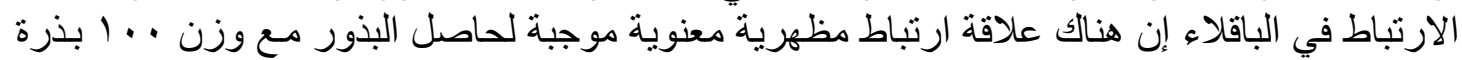

. $(r$. Thalib و Talal)

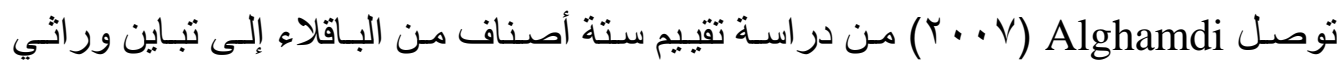

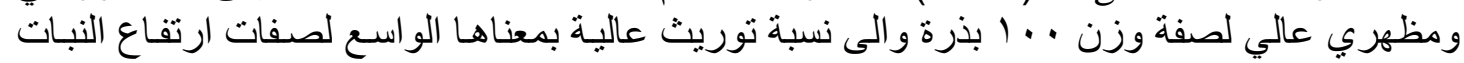

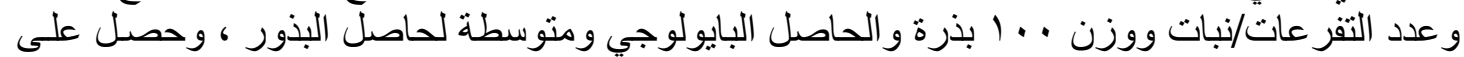

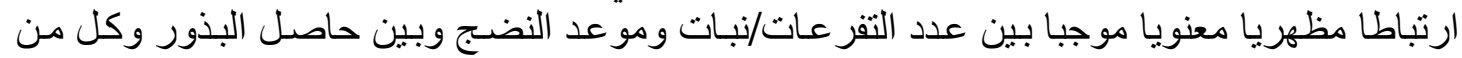

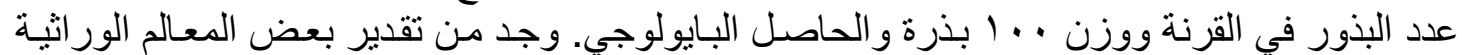

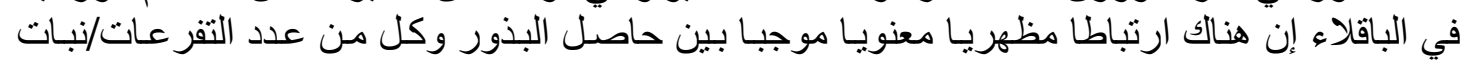

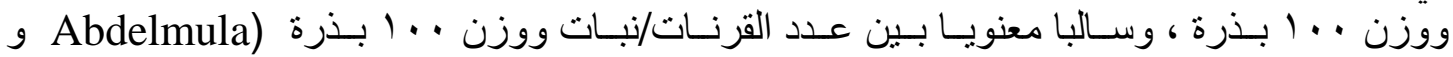

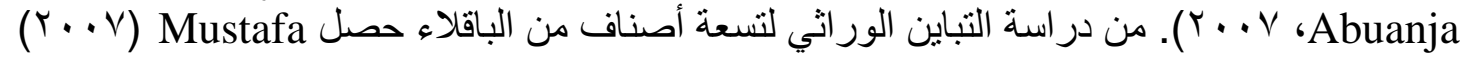

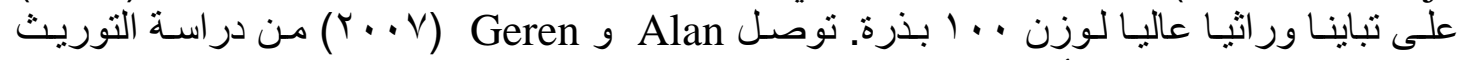

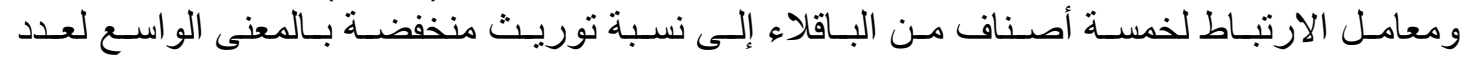

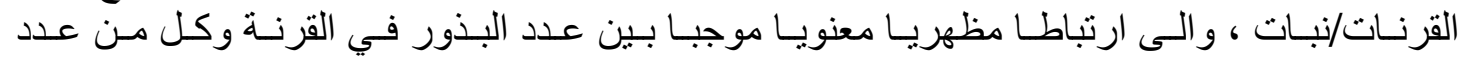

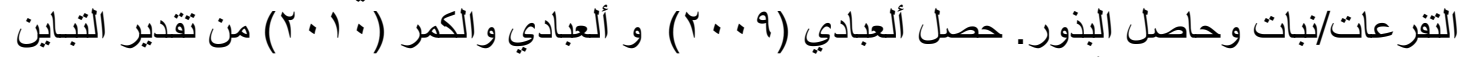

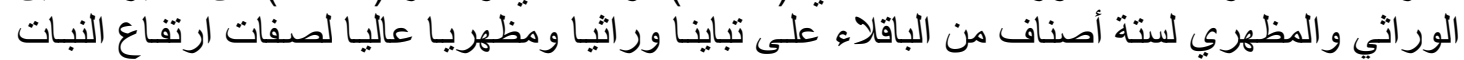

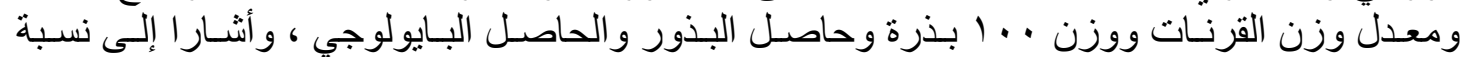

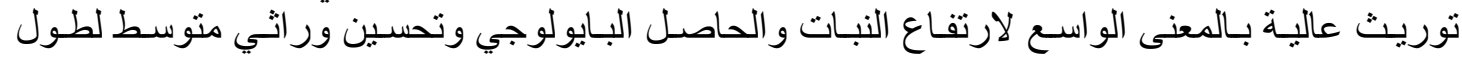

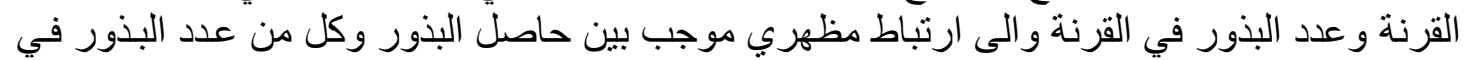

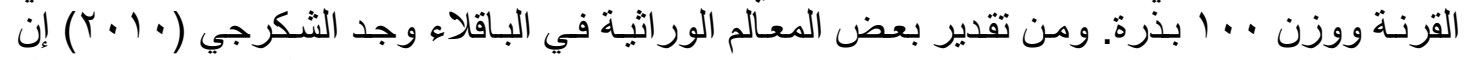

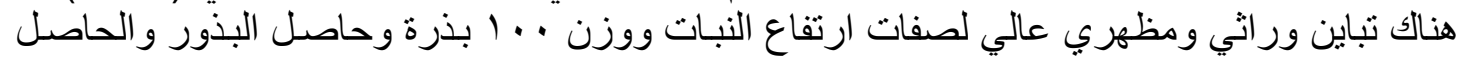

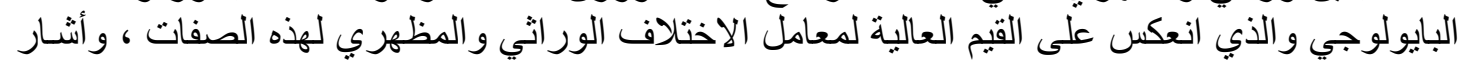

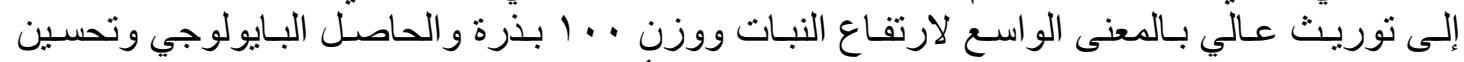

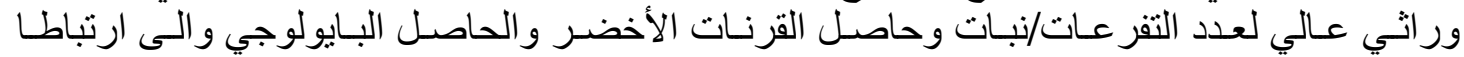

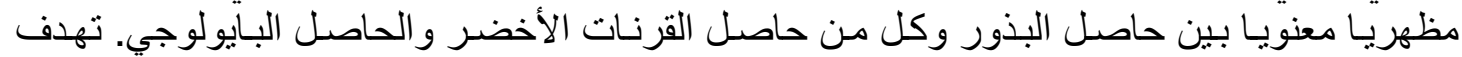

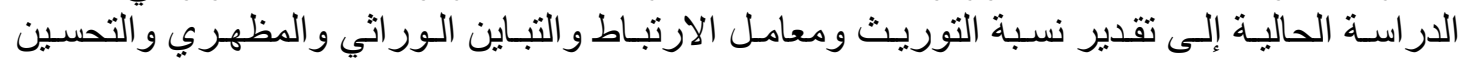

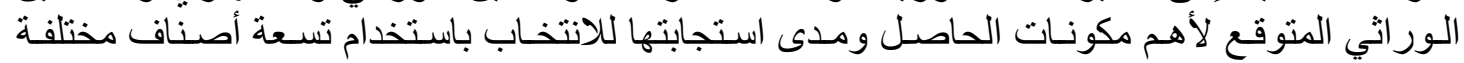
المصدر من الباقلاء للاستفادة منها في بر امج تربية وتحسين هذا لهابت المحصول. 


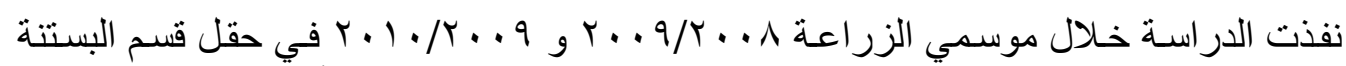

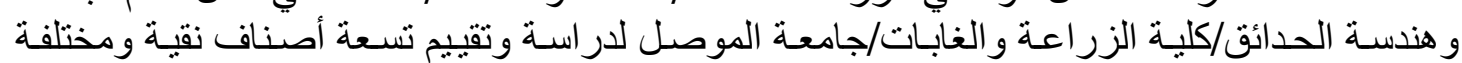

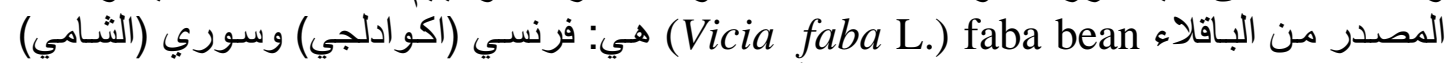

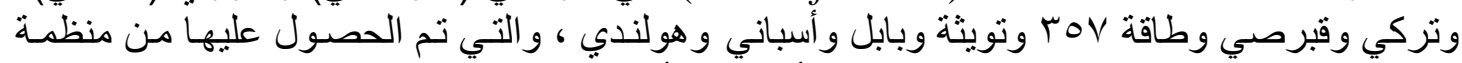

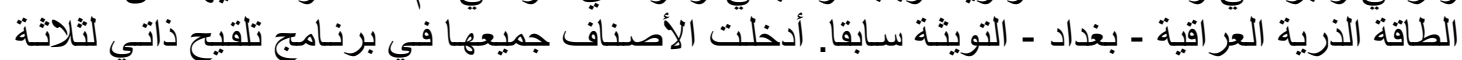

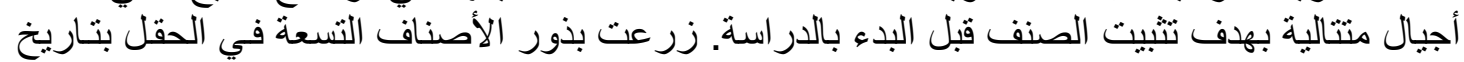

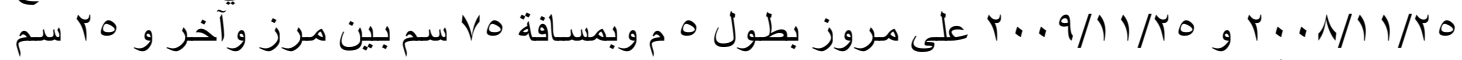

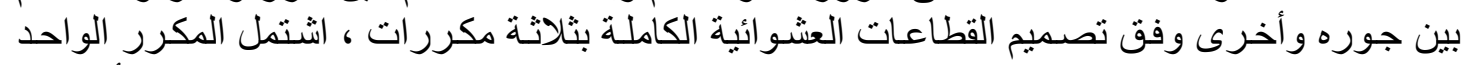

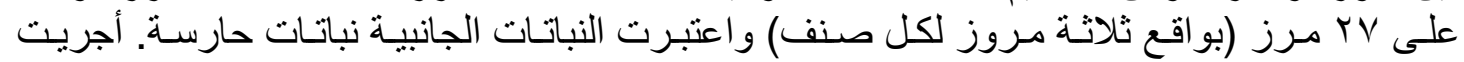

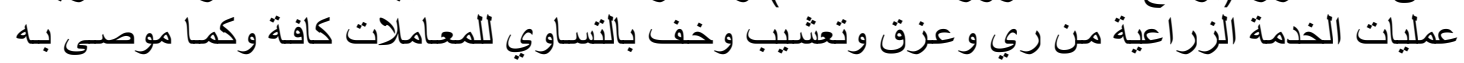

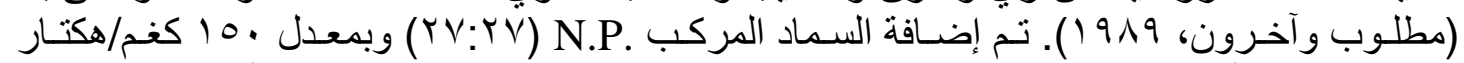

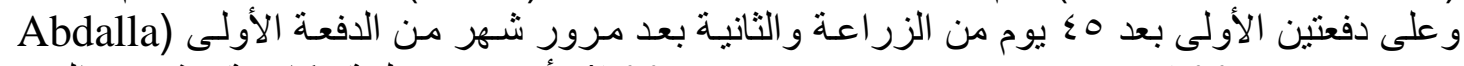

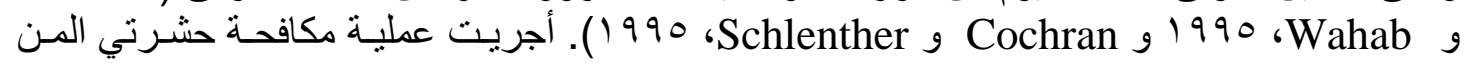

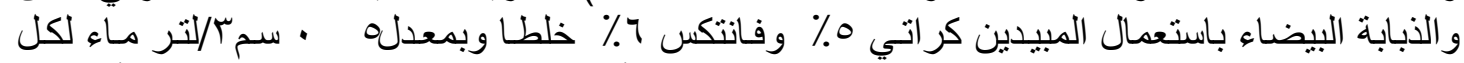

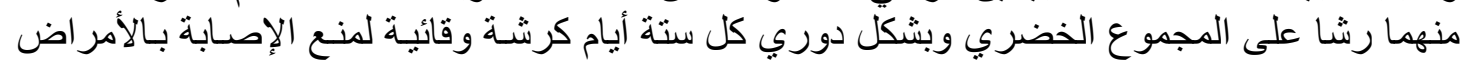

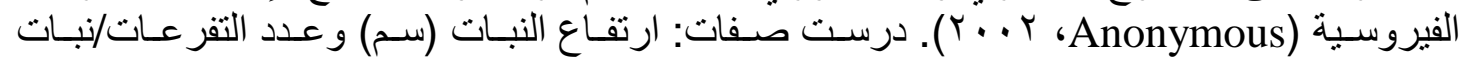

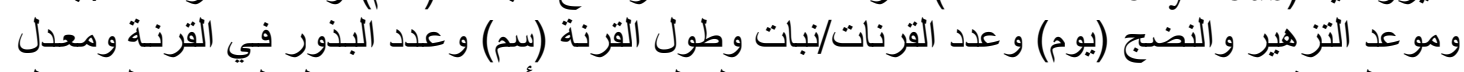

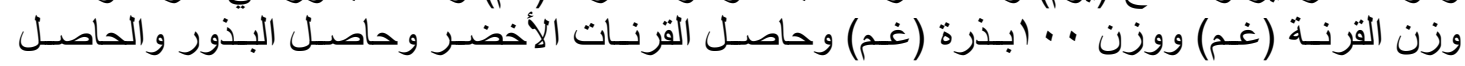

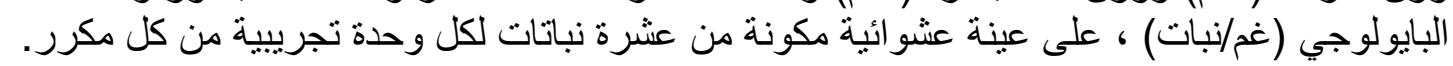

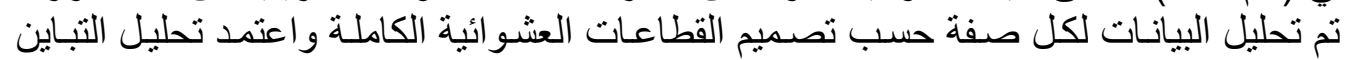

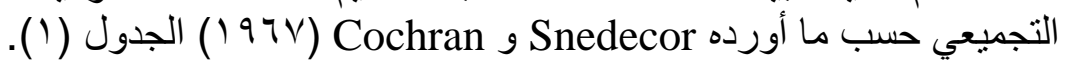

\begin{tabular}{|c|c|c|c|}
\hline S.O.V. & d.f. & M.S. & E.M.S. \\
\hline $\begin{array}{c}\text { Years } \\
\text { Rep / Years } \\
\text { Varieties } \\
\text { Varieties } \times \text { Years } \\
\text { Error }\end{array}$ & $\begin{array}{c}\mathrm{Y}-1=1 \\
\mathrm{Y}(\mathrm{r}-1)=4 \\
(\mathrm{~V}-1)=8 \\
(\mathrm{~V}-1)(\mathrm{Y}-1)=8 \\
\mathrm{Y}(\mathrm{r}-1)(\mathrm{V}-1)=32\end{array}$ & $\begin{array}{l}\text { M1 } \\
\text { M2 } \\
\text { M3 }\end{array}$ & $\begin{array}{c}\sigma^{2} \mathrm{e}+\mathrm{r} \sigma^{2} \text { ay }+ \text { ry } \sigma^{2} \mathrm{a} \\
\sigma^{2} \mathrm{e}+\mathrm{r} \sigma^{2} \text { ay } \\
\sigma^{2} \mathrm{e}\end{array}$ \\
\hline
\end{tabular}

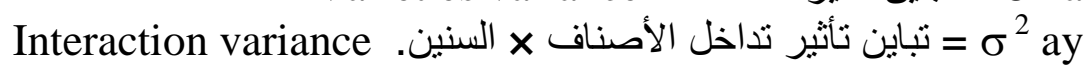

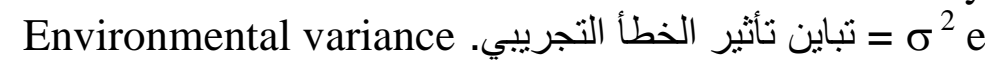

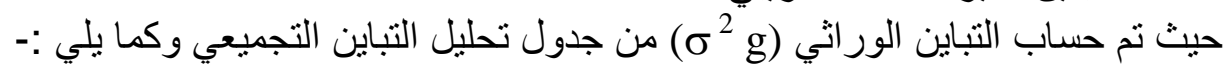
$\sigma^{2} \mathrm{~g}=(\mathrm{M} 1-\mathrm{M} 2) / \mathrm{ry}$

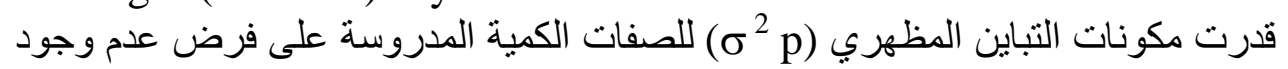

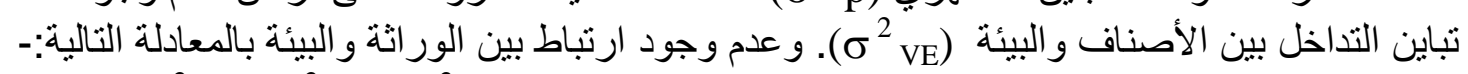

$$
\begin{aligned}
& \sigma^{2} \mathrm{p}=\sigma^{2} \mathrm{~g}+\sigma^{2} \mathrm{e} \\
& \sigma^{2} \mathrm{e}=\mathrm{M} 3 \\
& \text { وقدر معامل الاختلاف الور اثي (GCV) و المظهري (PCV) باستخدام المعادلات الآتية:- } \\
& \mathrm{GCV} \%=\left(\sqrt{ } \sigma^{2} \mathrm{~g} / \tilde{\mathrm{Y}}\right) \times 100 \text {. } \\
& \operatorname{PCV} \%=\left(\sqrt{ } \sigma^{2} \mathrm{p} / \tilde{\mathrm{Y}}\right) \times 100
\end{aligned}
$$


$\mathrm{h}_{\text {b.s. }}^{2}=\left(\sigma^{2} \mathrm{~g} / \sigma^{2} \mathrm{p}\right) \times 100$.

وحسبت نسبة التوريث بالمعنى الواسع كما يلي:-

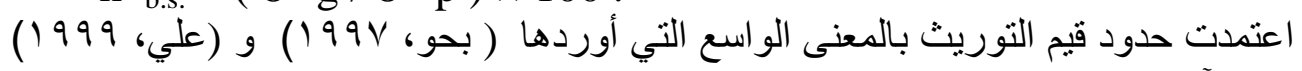

(الآتي.

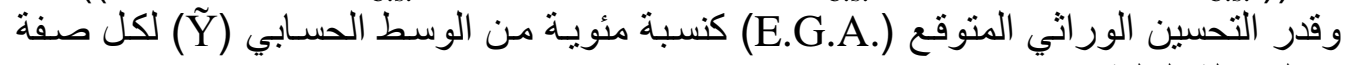

عن طريق المعادلة التالية:-
E.G.A. $\%=\left[\left(\mathrm{K} \mathrm{h}^{2}{ }_{\text {b.s. }} \sqrt{ } \sigma^{2} \mathrm{p}\right) / \tilde{\mathrm{Y}}\right] \times 100$.

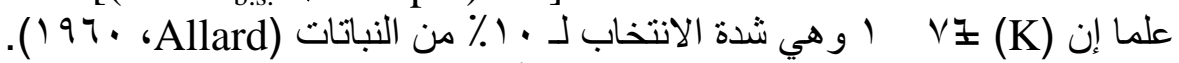

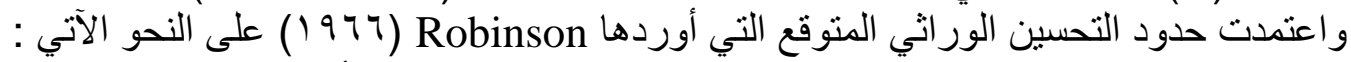

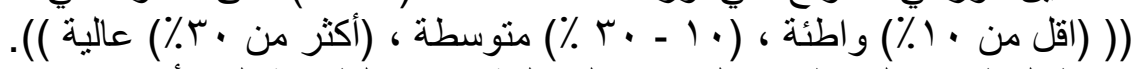

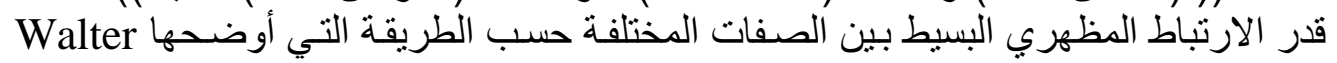

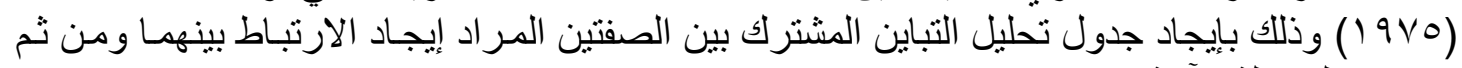
استخدام المعادلة الآتية : (1وليجاد جدو

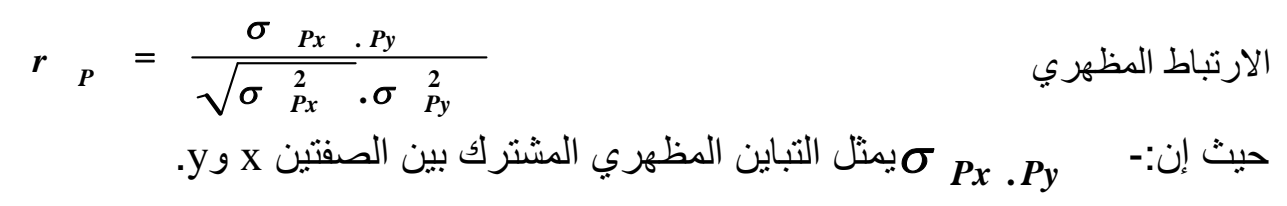

\footnotetext{
النتائج والمناقشة إنائة

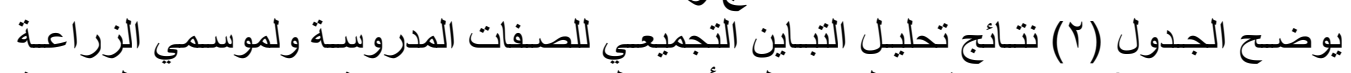

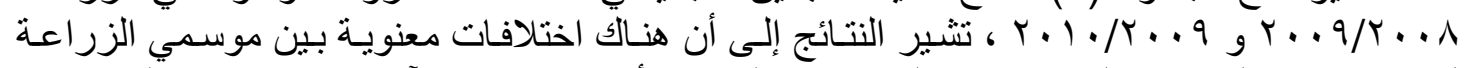

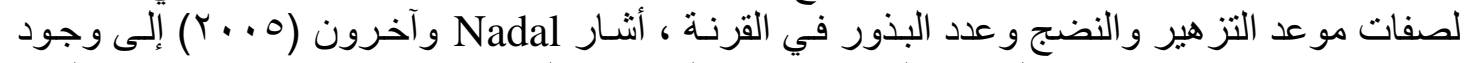

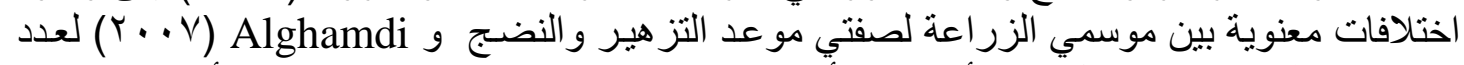

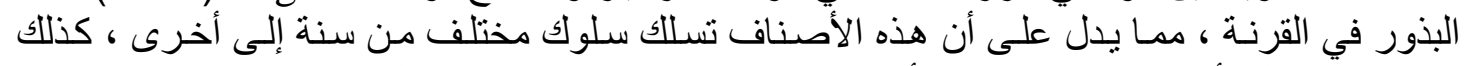

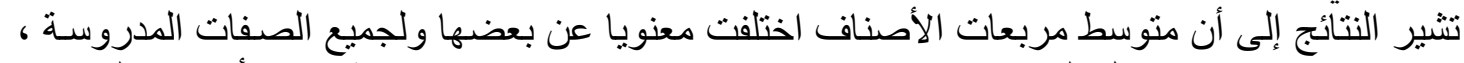

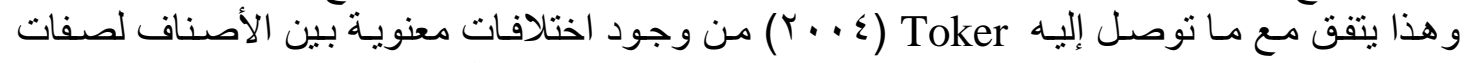

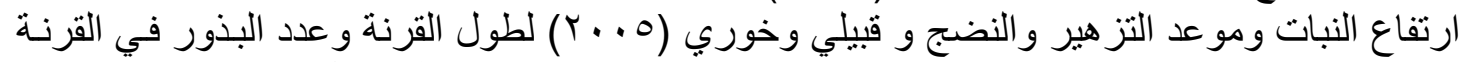
و Alghamdi و و Geren

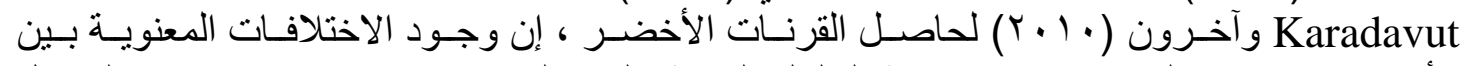
الأصناف ضروري للاستمر ار في در اسة السلوك الور الوراثي لهذه الصفات بهدف تحسينها. كان التداخل بين الأصناف × السنين معنويا لصنفة لاصني
} 


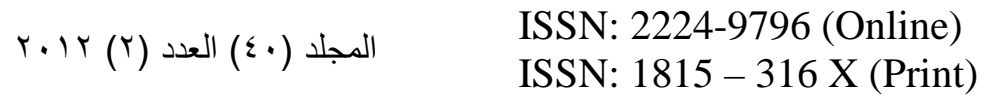

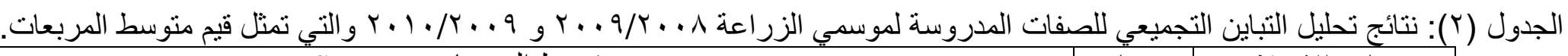

\begin{tabular}{|c|c|c|c|c|c|c|c|}
\hline \multicolumn{6}{|c|}{ Mean Squares متوسط المربعات } & \multirow{2}{*}{ الحرية } & \multirow[t]{2}{*}{ مصنادر الاختلاف } \\
\hline 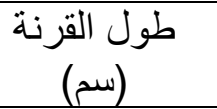 & عدد القرنات & موعد النضـج & مو عد التز هير & عدد التفرعات & $\begin{array}{c}\text { ارتفاع النبات) } \\
\text { (سم) }\end{array}$ & & \\
\hline$\Gamma \leqslant \wedge \wedge$. & r oov & $* * \vee \circ 9 \quad \ldots 1$ & $* \star \varepsilon r V \quad \cdots V$ & . OYY & or $\quad \wedge \leqslant 7$ & 1 & السنين \\
\hline $77 \cdot r$ & *Yo & IYO 9VI & $\varepsilon \quad 7 \leqslant \wedge$ & *T TVE & $* * Y r q \quad . r \leq$ & $\varepsilon$ & المكرر ات / السنين \\
\hline 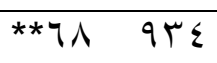 & $* * 01 \quad r 70$ & $*{ }^{*} 1 \cdot 1 \cdot 1$ & $* 1 \leqslant \vee \quad 99 \wedge$ & **Y। $\quad 9 \leqslant V$ & **ITVR 909 & $\wedge$ & الأصناف \\
\hline *To $17 \varepsilon$ & $.9 r$ & $I Y \leq \quad A \cdot r$ & $1 \ldots V \vee 1$ & 0.1 & r7o & $\Lambda$ & الأصناف × السنين \\
\hline I $\leqslant \wedge$. & OrI & $9 V Y$ & $O \wedge V \wedge I$ & $r \cdot 1$ & $V \mu V$ & rr & الخطأ التجريبي \\
\hline
\end{tabular}

\begin{tabular}{|c|c|c|c|c|c|c|c|}
\hline \multicolumn{6}{|c|}{ منوسط المربعات Mean Squares } & \multirow{3}{*}{ درجاتة } & \multirow[t]{2}{*}{ مصادر الاختلاف } \\
\hline (غاصل البايولوجي & (غاصلن البذور & الأخضر (غاصل القرنات) & وزن (غ) (بذرة & القرنة (غم) وزن & عدد البذور في & & \\
\hline TIAqY VVA & .701 & lvrqr $0 \leq 9$ & $r \cdot \Lambda \leqslant r V$ & $9 V \quad Y \wedge \varepsilon$ & $* * 1, \quad \wedge \wedge 1$ & & السنين \\
\hline DITH ITH & **ร19 $11 N$ & EMVO OrA & $1 V 9 \quad 1 \leqslant V$ & rAY $\Xi I \Sigma$ & r TVA & $\varepsilon$ & المكرر ات / السنين \\
\hline$* * 1 V 1 V \cdot 1 \quad 109$ & **VYฯ Mा & **orovo 0.9 & $* * 1 \vee \vee 7 \quad \ldots 1$ & $* * 7 \leqslant 0 \quad 00$. & * $\quad$ rq & $\wedge$ & الأصناف \\
\hline$\Gamma \leqslant 1 \leqslant \wedge . \varepsilon$ & 7r 19. & r^ol $\quad T \leq r$ & $1 \wedge \leqslant \quad \cdot \leq 0$ & $1 \vee \wedge \quad 9 \wedge 9$ & $\cdots 1$ & $\wedge$ & الأصناف × السنين \\
\hline IY..T TrY & Ar $\cdot$ VI & $11 \leqslant \vee \wedge \quad \wedge .7$ & $11007 \mathrm{~V}$ & $1 \wedge 1,1.1$ & I YIV & rT & الخطأ التجريبي \\
\hline
\end{tabular}


طول القرنة عند مستوى احتمال ه\%. هذه النتائج تشير إلى إن الأصناف قد سلكت سلوك متباين بين البيئات المختلفة (بيئتين).

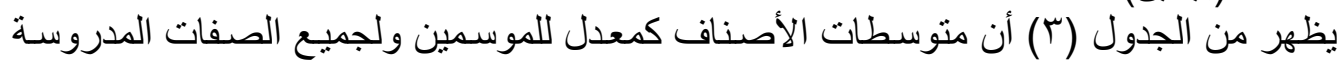

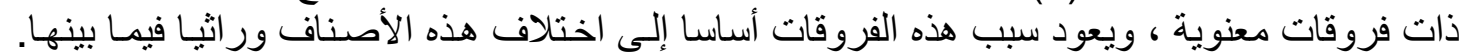

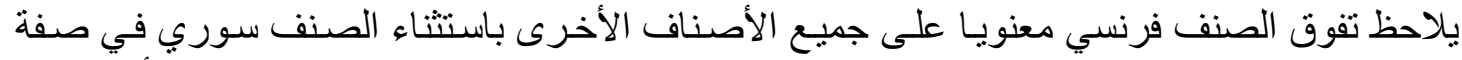

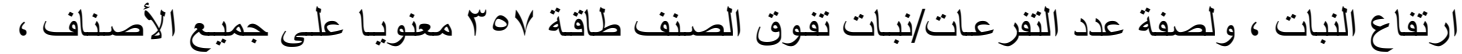

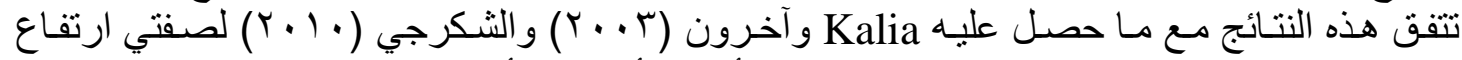

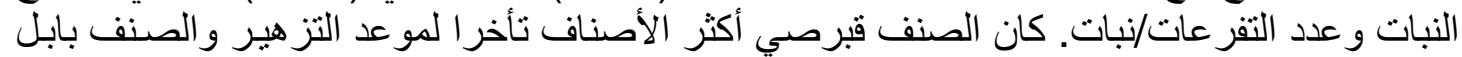

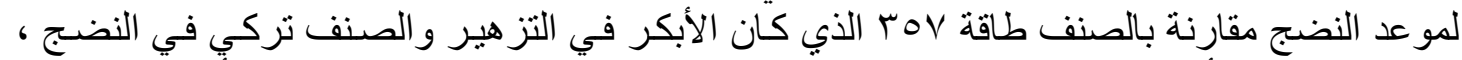

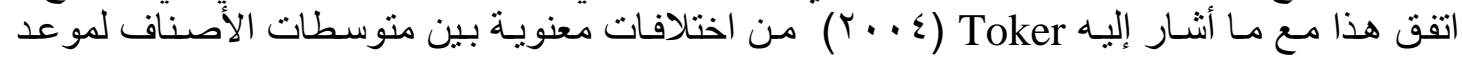

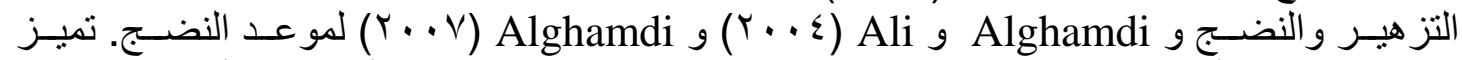

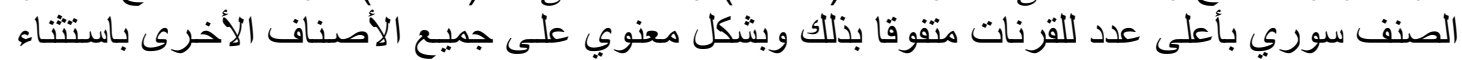

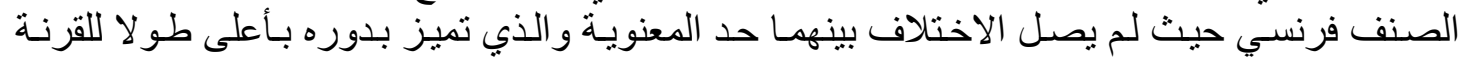

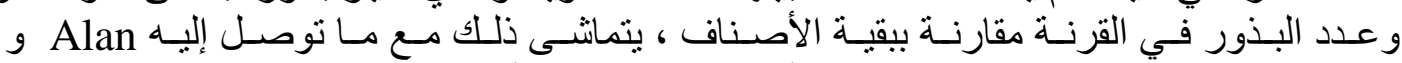
Geren

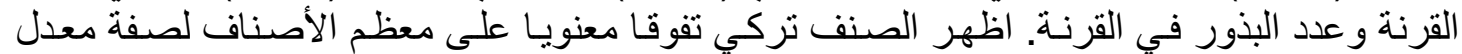

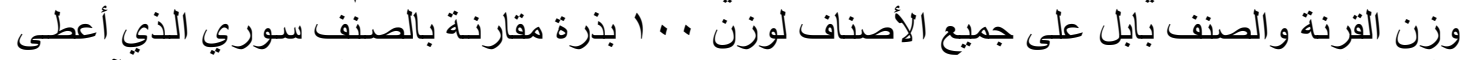

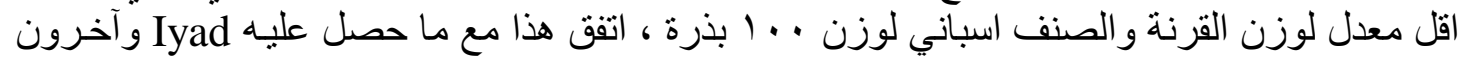

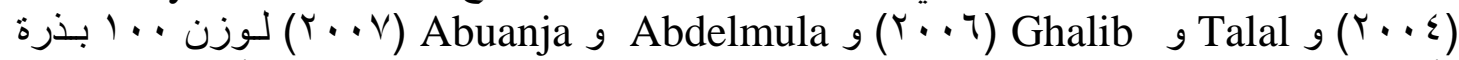

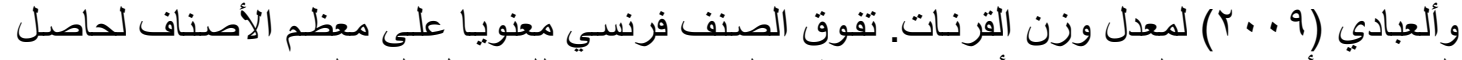

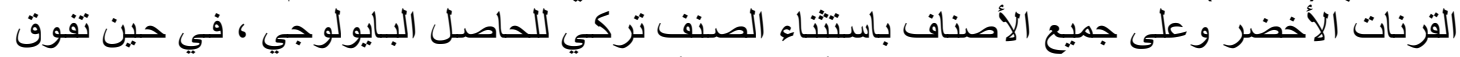

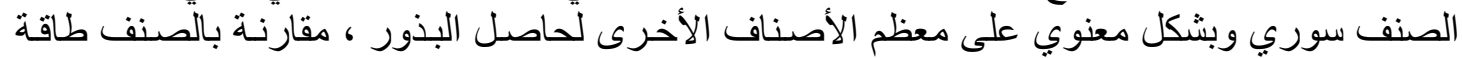

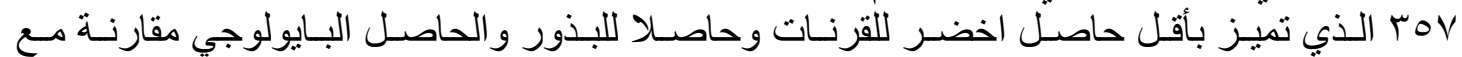

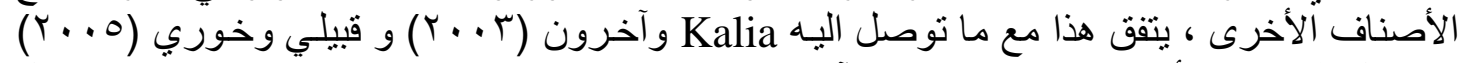

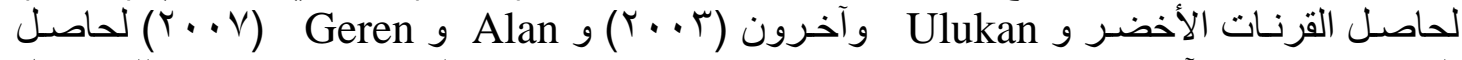

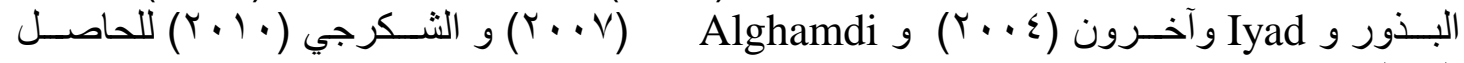

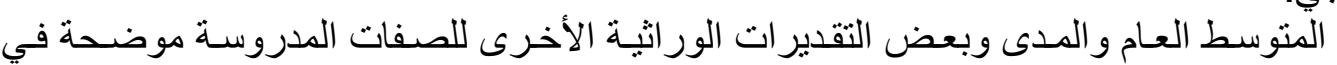

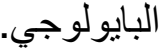

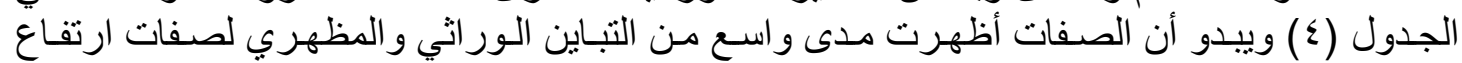

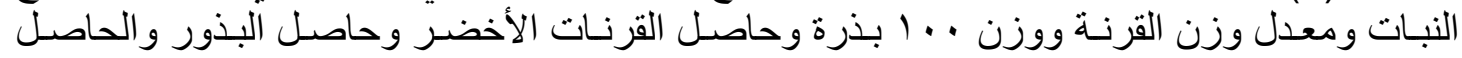

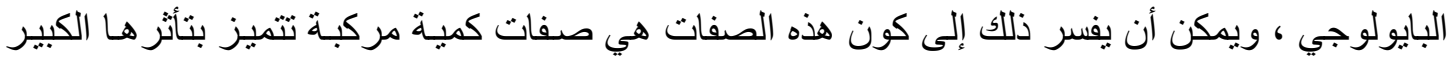

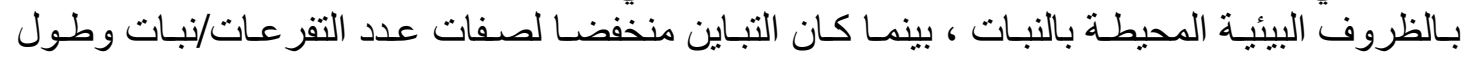

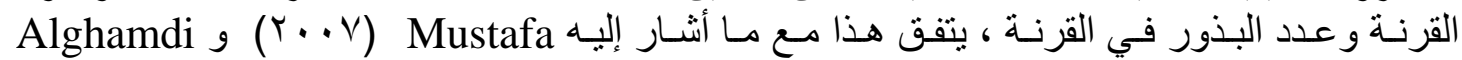

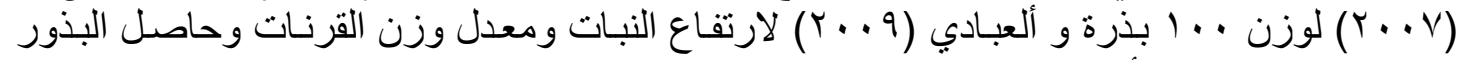

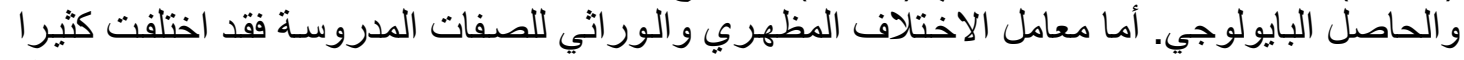

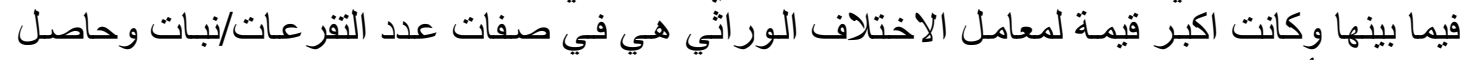

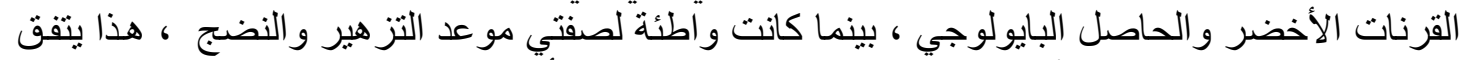

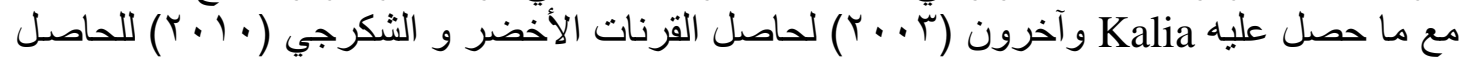

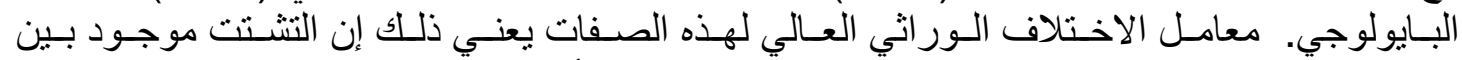

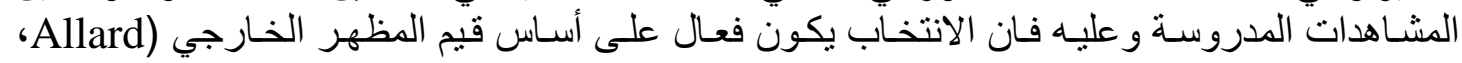

. (1910، Chaudhary و Singh و 197.

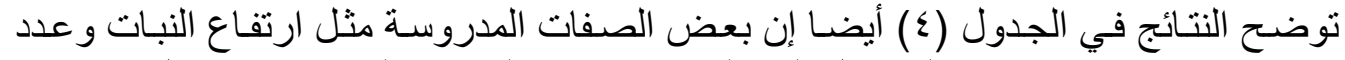

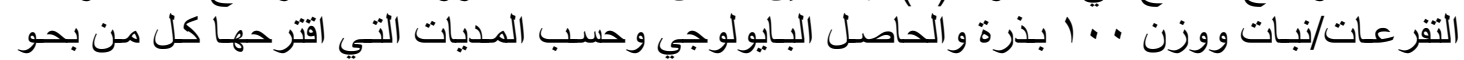

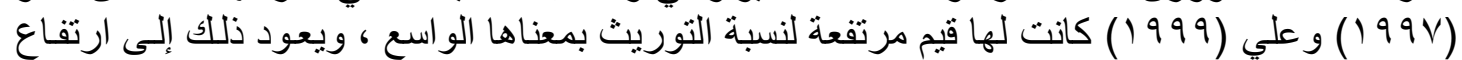

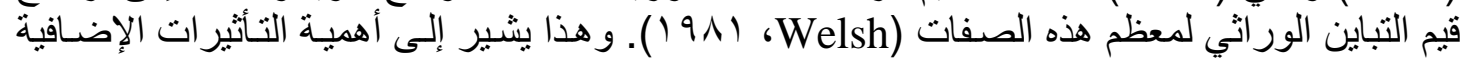




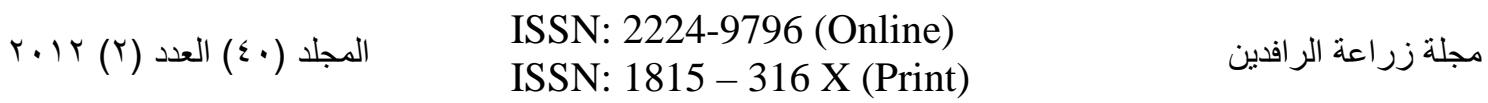

$$
\begin{aligned}
& \text { و غير الإضـافية للجينـات التي تسبطر على وراثـة هذه الصـفات (Mather و Jinks، برو ( ). إن } \\
& \text { ارتفاع نسبة التوريث }
\end{aligned}
$$




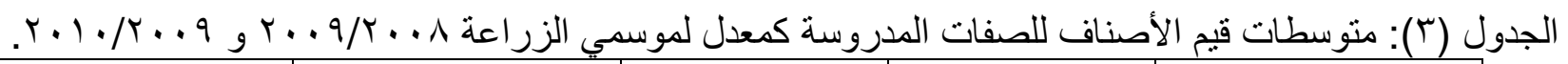

\begin{tabular}{|c|c|c|c|c|c|c|}
\hline طول القرنة سم) & ع عدد القرنات & موعد النضج & مو عد التز هير & عدد التفر عات & $\begin{array}{c}\text { ارتفاع النبات } \\
\text { (سم) }\end{array}$ & الصفات المدروسة \\
\hline I ro $0 \leqslant 1$ & rا & V r r & ج- I91 170 & سب ل Vب & $i v \varepsilon$ & فرنسي (اكو ادلجي) \\
\hline . r ب ج & il 1 9人 & i IVo & rأب & 7 ب ج & ا ل أ ب & سوري (الثامي) \\
\hline ج11 701 & |r V V V V & 109 ب ب & ج- أ & | 11 ا 7 ب - د & " & تركي \\
\hline ا ج & & IIVE NYo & II. $\mathrm{r}$ NIV & $د \leq \wedge \vee 1$ & دOr & قبرصي \\
\hline 10 & ج $\leqslant$ rqV & rی . & ( & 11.10. & gr. & طاقة rov \\
\hline 19 & & IIVE Vo. & ج- أ 9090 & اו & & تويثة \\
\hline 19 & اب اب & $11 \mathrm{VV} \quad 11 \mathrm{~V}$ & $i 1 . r \quad \sigma$. & ج & د 09 9 91. & بابل \\
\hline 10 & 1 & & ج-أ 9६ 99. & ه 101 & $\rightarrow \leqslant \varepsilon$ & اسباني \\
\hline r l r r ro & $T \leqslant Y$ & & و & ه r r r . & $\rightarrow \leq 1$ & هولندي \\
\hline
\end{tabular}

\begin{tabular}{|c|c|c|c|c|c|c|}
\hline الحاصل البايولوجي & حاصل البذور & الأخضر (غاصل القرنات & وزن · · (بذرة (غم) & القرنة (غدل وزن & في القرنة البذور & الصفات المدروسة \\
\hline INYO VTI & Iov ov. & $1 \leq 0 . \quad 171$ & 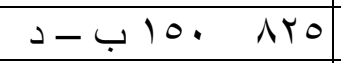 & أب & $17 r . r$ & فرنسي (اكو ادلجي) \\
\hline ب OVA $\varepsilon 9 r$ & TYA & ج-IKr. Ir. & & Tr 001 & أب & سوري (الثامي) \\
\hline IVTr & أ & r & $ه-ج \mid \leq 1 \quad \vee q \mu$ & $1007 \%$. & ع ب ج & تركي \\
\hline & 107910 & & ه & & ع ب ج & قبرصي \\
\hline د rOq & $د T V$ & D & $ه-1 \leqslant 7 \leqslant \Lambda$. & & T V90 & طاقة rov \\
\hline . & أب & ج & 109 V9V & $ج-I \mu \wedge \quad V \leqslant r$ & 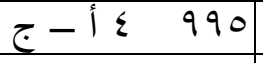 & تويثة \\
\hline 1 & 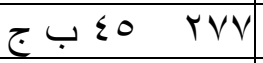 & 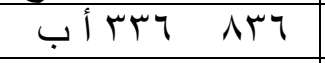 & IIVV NKr & r rov & ج & بابل \\
\hline Tr TY Tr & 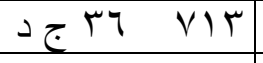 & 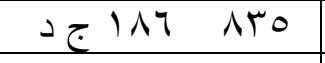 & 9110 NRr & $ج \quad r \leqslant \quad r \leqslant V$ & & اسباني \\
\hline ج & 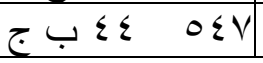 & דr 1 1 . & תו & ج & S Y V & هولندي \\
\hline
\end{tabular}

الجدول (ء): المتوسط العام ومعامل الاختلاف و التحسين الور اثي المتوقع للحاصل ومكوناته في الباقلاء. 


\section{～ISSN: 2224-9796 (Online)}

ISSN: 1815 - $316 \mathrm{X}$ (Print)
مجلة زر اعة الر افدين

\begin{tabular}{|c|c|c|c|c|c|c|c|c|c|c|c|c|c|}
\hline \multicolumn{2}{|c|}{ التحسين } & \multicolumn{2}{|c|}{ التوريث } & \multicolumn{2}{|c|}{ الاختلاف المظهري } & \multicolumn{2}{|c|}{ الاختلاف الوراثي } & المظهرين التباين & الوراثي & \multicolumn{2}{|r|}{ المدى } & والانحر اف القياسي العام & الصفات المدروسة \\
\hline$\varepsilon \cdot$ & rrTt & $\vee \wedge$ & Ort & rq & 1.9 & ro & V9Y & ro9 711 & $r \cdot r \quad q \leqslant q$ & $V \varepsilon$ & $70 Y r$. & . $\quad \wedge r \# 00 \quad r 09$ & ارتفاع النبات (سم) \\
\hline$\varepsilon V$ & ONY & $V \varepsilon$ & $\Lambda \leqslant N$ & T & IrE & M & ror & $\varepsilon \vee \vee \vee 0$ & r ov & 1 . & $10-r r q$. & $10 \cdot r \# 7 \cdot \leq 9$ & عدد التفر عات/ نبات \\
\hline 1 & Voo & 11 & 1.9 & $\wedge$ & $\varepsilon \leqslant 0$ & $r$ & $9 \cdot Y$ & $7770 \%$ & $\vee \wedge \vee 1$ & $1 \cdot r$ & $\wedge \backslash \star \wedge \wedge \quad r \ldots$ & $r \quad \cdots \pm 97 \quad 77 r$ & مو عد التز هير (يوم) \\
\hline 1 & $\varepsilon \pi$ & $1 \pi$ & rov & 7 & .19 & r & rTA & $1 . V \quad r .0$ & سח & IVV & $11 * 109 \quad 114$ & $0 \quad 70 \underline{1} 179 \quad 19 \mathrm{~V}$ & موعد النضج (يوم) \\
\hline rq & $r v-$ & rᄉ & 190 & $\varepsilon r$ & $9 \leqslant Y$ & $r 7$ & $V \vee \varepsilon$ & IV YYT & 7790 & $1 \varepsilon$ & $q \wedge E \leq \quad r q V$ & $7 V 1$ 斗9 775 & عدد القرنات/نبات \\
\hline 11 & $1 \wedge 7$ & YV & OYT & $r$ r & .94 & Ir & 110 & $r \cdot \quad \varepsilon \leqslant \Lambda$ & O TYA & ro & $0 \leqslant A \quad 10 \leqslant 90$ & $r \quad$ ov $\underline{z} 19$ & طول القرنة (سم) \\
\hline 11 & $r \cdot \varepsilon$ & rT & $1 \wedge 9$ & $r 7$ & $7 \leq 9$ & 11 &. ro $^{2}$ & 1099 & rAr & 7 & Y.Fr V9O & $r \quad T \leq \underline{\#} \varepsilon$ & عدد البذور في القرنة \\
\hline$r \varepsilon$ & rAV & rq & $r \leqslant Y$ & $\sum V$ & $1 \wedge \varepsilon$ & Yo & 011 & Y70 & $V V \quad V 7$. & 00 & $T Y-Y M \quad 001$ & $9 \leqslant \# r \quad 00 \mathrm{~V}$ & معدل وزن القرنة (غم) \\
\hline 17 & $r \wedge 1$ & 79 & 701 & 11 & r^T & 11 & .199 & rᄉ. $\quad \wedge 9 r$ & rTO RYT & IVV & NTE $110 \quad$ NTr & .7 年 $1 \leq 7 \quad \wedge 9$. & وزن · · (بذرة (غم) \\
\hline ru & 7.0 & $\leqslant r$ & हार & $\leqslant 9$ & .49 & M & $94 \mathrm{y}$ & 1994T VAT & ᄉ & $\leqslant 0$. & $17+110$ & $1 \wedge \quad 11 \#$ YAV NA & حاصل القرنات الأخضر (غم/نبات) \\
\hline TA & $9 \leq Y$ & ov & $\sum Y Y$ & $r \wedge$ & $7 r y$ & YI & $v \cdot 1$ & $194 \quad$ VOV & 11.711 & 09 & TYA YV . TA & $1.9 \quad r 7 \underline{E} \leqslant 1 \leqslant 1$. & حاصل البذور (غم/نبات) \\
\hline$\leqslant 0$ & sor & $\checkmark \cdot$ &.$r_{0}$ & Tr & $\wedge \wedge$. & $r \cdot$ & ᄉтI & $\{\ldots 00.01 \leqslant$ & $r \wedge \cdot \leq \Lambda \quad \wedge q r$ & NYo & $V 7+r 09$ rrs & IT ITE & الحاصل البايولوجي (غم/نبات) \\
\hline
\end{tabular}


تدل على إمكانية إدخال تحسينات مباشرة على هذه الصفات في السنين التالية من خـلال بر امج الته التربية

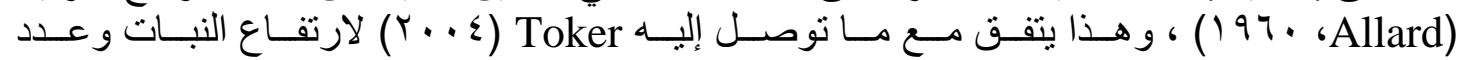

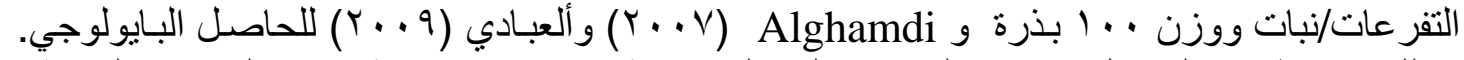

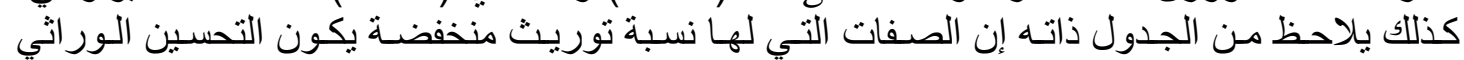

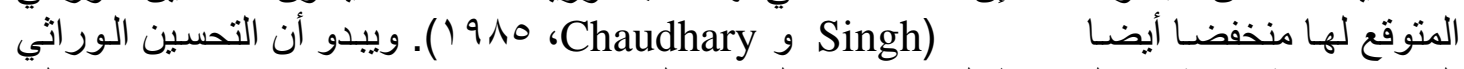

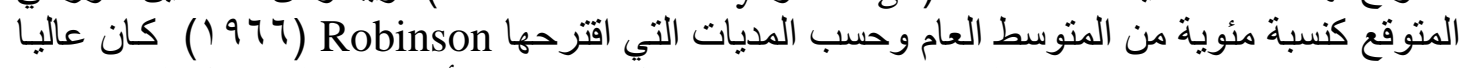

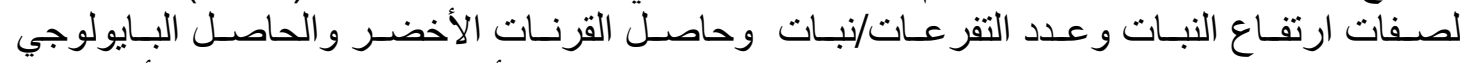

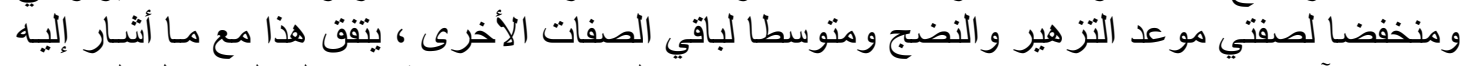
Kalia

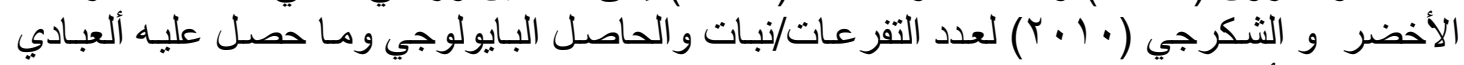

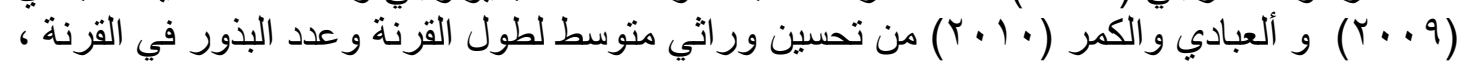

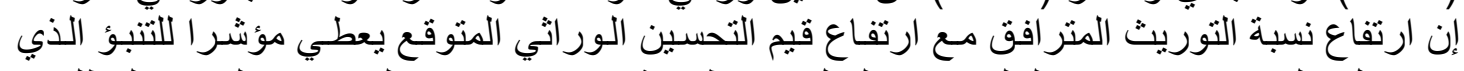

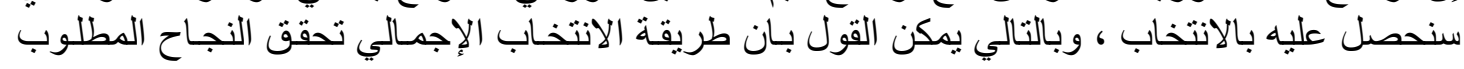
.( (191)،Welsh)

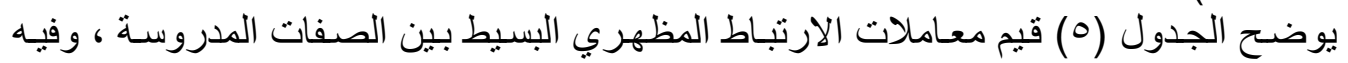

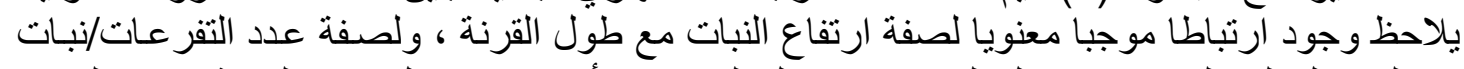

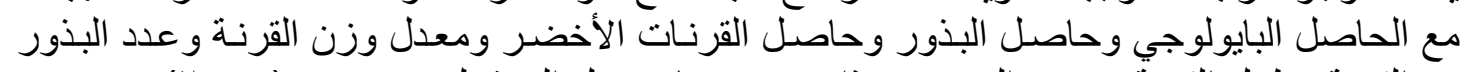

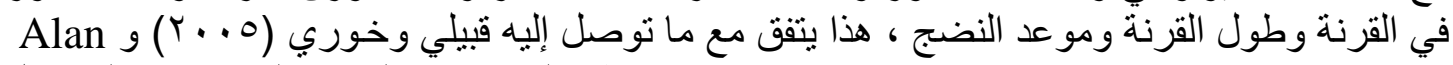

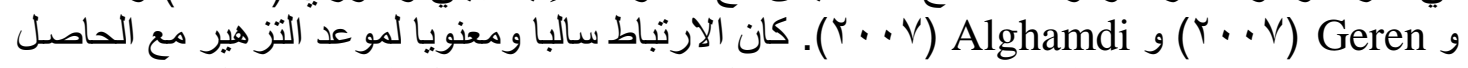

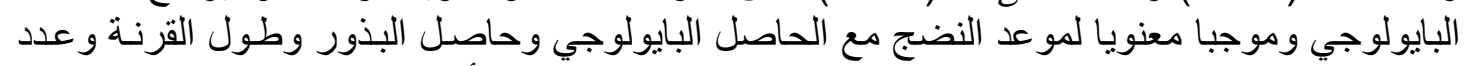

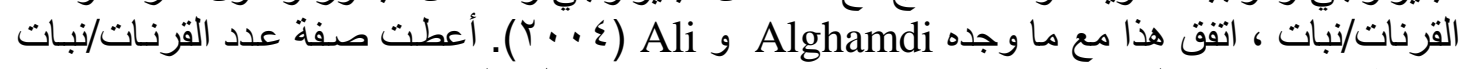

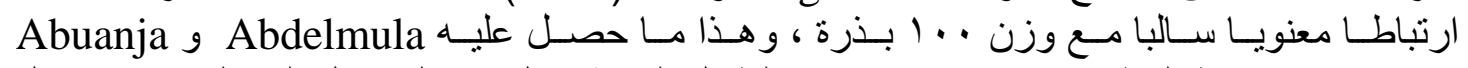

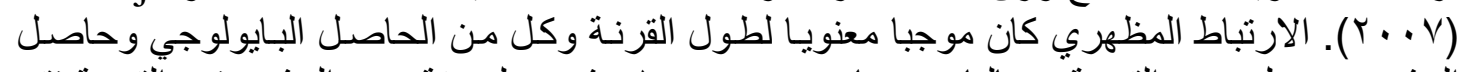

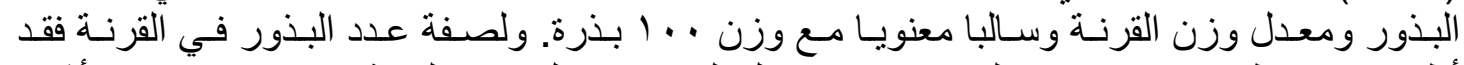

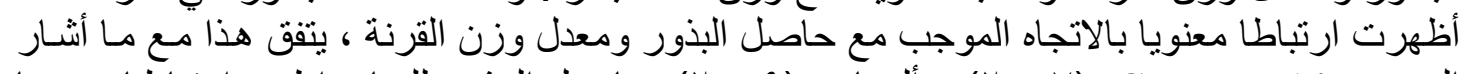

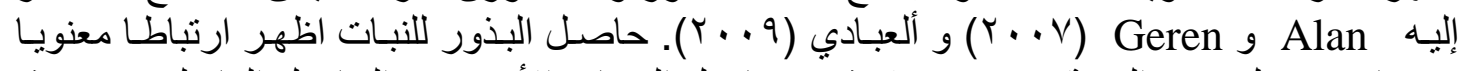

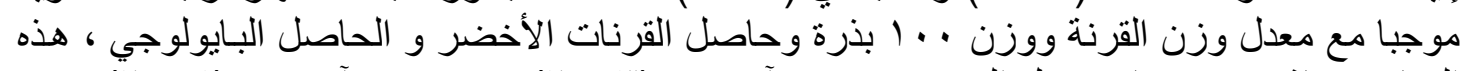

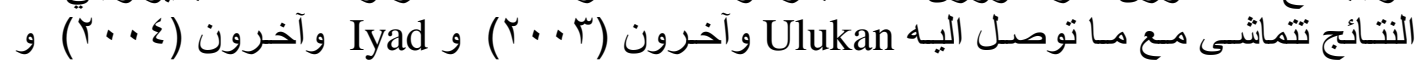

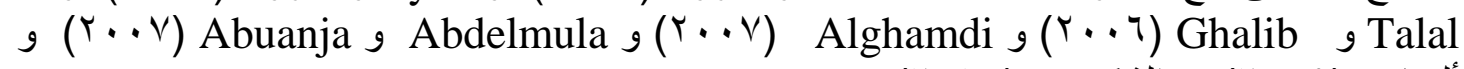

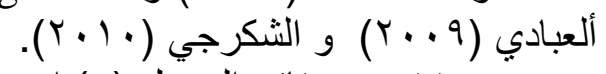

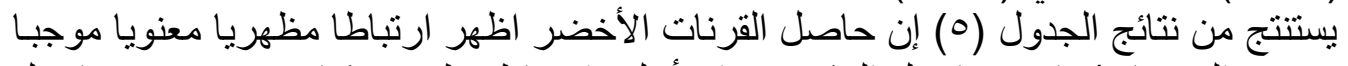

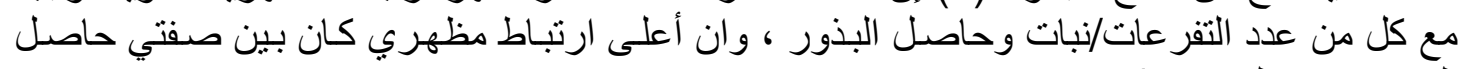
البذور و عدد التفر عات/نبات. 
الجدول (0): معاملات الارتباط المظهري البسيط بين الصفات المدروسة.

\begin{tabular}{|c|c|c|c|c|c|c|c|c|c|c|c|}
\hline التفر عاد & التز هو عد & مو عد النضج (يوم) & القرنات & طولة & البذور في القرنة & معدل وزن & ، . . بذرة & الأخضرنات & (غم/نباصت & البايولوجي الحاصلي & الصفات المدروسة \\
\hline \multirow[t]{11}{*}{17} & .101 & - $\leqslant$ & $\cdot \quad 1 \leq \cdot$ & *. $\mu V T$ & . $11-9$ &.$V T$ & $\cdot \mu \cdot \Lambda$ & . IM-7 & . . . & - $\quad$ Yl-9 & ارتفاع النبات (سم) \\
\hline & $\cdot \quad \cdot 5-7$ & **, 099 &.$\quad 19 \pi$ & **. TVV & *. भนN & $* * . \quad 0 \wedge y$ & $\cdot . V \varepsilon$ & *. TOT & $* *, \quad \vee 19$ & $* * . \quad 0 \wedge$. & عدد التفر عات/ نبات \\
\hline & & - IM &.$\quad .0 .9$ & - IT-A & - $\quad T \leq-r$ & .09 & . $\quad K V I$ & . $\quad \mathrm{MIV}$ & $\cdot \quad \cdot \Sigma \cdot$ & *. $\leqslant 1-7$ & مو عد التز هير (يوم) \\
\hline & & & *. Mtr & *. $\leqslant Y \wedge$ &.$\quad .10$ & - $\quad r \cdot Y$ & .10. & . r97 & *. rol & **. $\leqslant 9 V$ & مو عد النضج (يوم) \\
\hline & & & & - MIV & . $12 \varepsilon$ & . $\quad Y 11$ & **, $\leqslant \wedge-\varepsilon$ & . TrO & $\cdot \cdot \cdot V-\varepsilon$ & $\ldots r$ & عدد القرنات/نبات \\
\hline & & & & & - TrE & $* * . \leqslant \Lambda$. & *. Tro & - $r 0 q$ & **. $\quad 0.9$ & *. MVI & طول القرنة (سم) \\
\hline & & & & & & $* *, \quad 7 \leqslant \mu$ & .1 .9 & - $r \wedge 9$ & ${ }^{* *}, \quad \leqslant \wedge 1$ & - TVA & عدد البذور في القرنة \\
\hline & & & & & & & $. \quad \cdot 1-0$ & $\cdot T \cdot V$ & *. $\quad \varepsilon \leqslant \varepsilon$ & . Irr & معدل وزن القرنة (غم) \\
\hline & & & & & & & & .1 .9 & **, 011 & . 1174 & وزن · · (بذرة (غم) \\
\hline & & & & & & & & & *. TVY & - $\quad r \leq 0$ & حاصل القرنات الأخضر (غم/نبات) \\
\hline & & & & & & & & & & $* *, \quad 019$ & حاصل البذور (غم/نبات) \\
\hline
\end{tabular}




\title{
PERFORMANCE, CORRELATION AND EXPECTED GENETIC ADVANCE FOR YIELD AND ITS COMPONENTS IN FABA BEAN (Vicia faba L.)
}

Shamil Y.Hassan AL-Hamdany

Dept. of Hort. \& Landscape Design, College of Agric. \& Forestry, Univ. of Mosul , Iraq

\begin{abstract}
The experiment was included evaluation of nine faba bean varieties: French (Aguadulce), Syrian (Shami), Turkish, Kobrisi, Tacka 357 , Tuweithe , Babylon, Spain and Holland and to determine genetic , phenotype variations , heritability, expected genetic advance and phenotypic correlation for yield and its components was carried out in the Field Dept. of Hort. \& Landscape Design, College of Agric. \& Forestry, Mosul University, during two growing season 2008/2009 and 2009/2010 by using Randomized Complete Block Design R.C.B.D. with three replications. Combined analysis results exhibited significant differences that the varieties between the two growing seasons for all the studied characters.

A significant varieties $x$ year interaction effect was found for pod length at $5 \%$. The results showed that the varieties were differed significantly for all the studied characters. French variety was highly superior for green pods yield and biological yield and Syrian variety for seed yield. There was a high genetic variation for plant height, average pod weight, 100 seed weight, green pods, seed and biological yield. High heritability for plant height, no. of branches per plant, 100 seed weight and biological yield, which means that most of the variation between varieties was due to genetic variations. High expected genetic advance for plant height, no. of branches per plant, green pods and biological yield. There was a high positive and significant correlation coefficient between green pods yield and no. of branches per plant and seed yield. The higher phenotypic correlations was found between seed yield and no. of branches per plant.

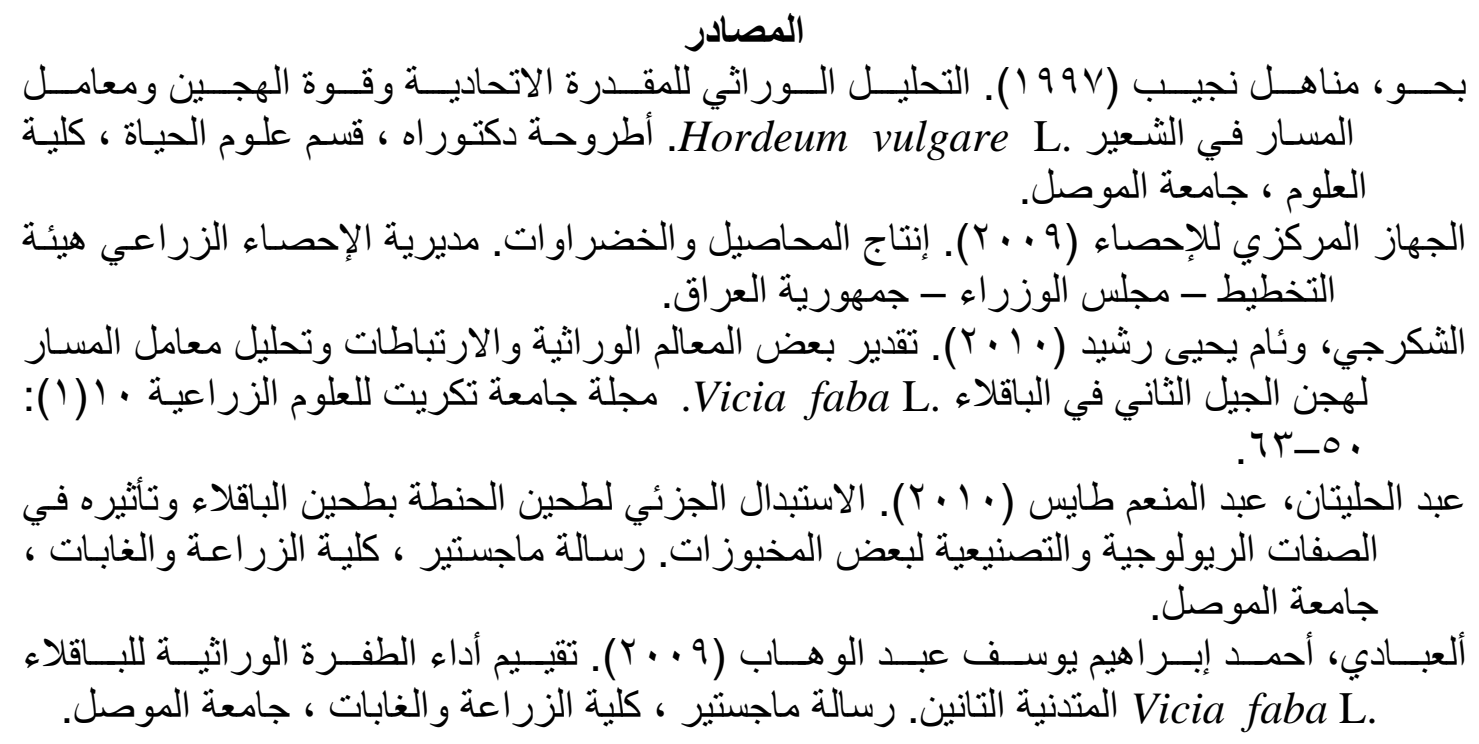




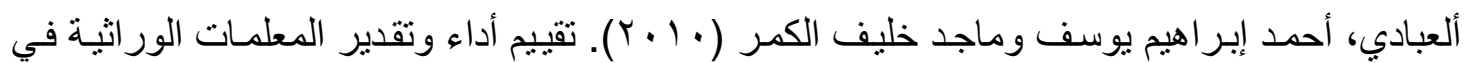

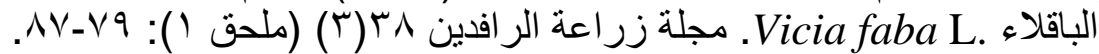

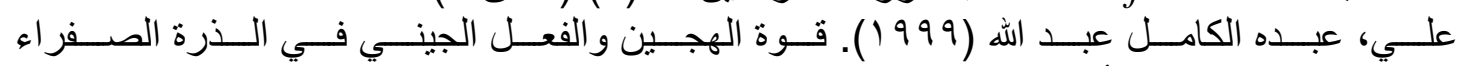
Zea mays L.

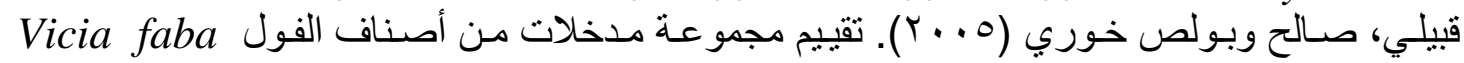

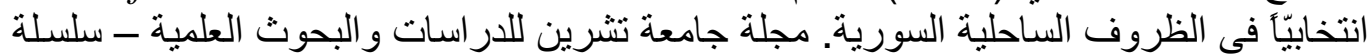

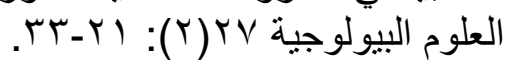

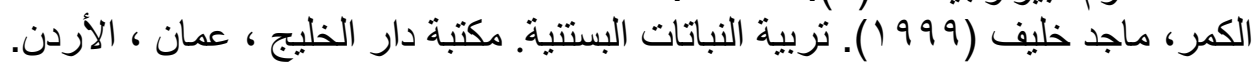

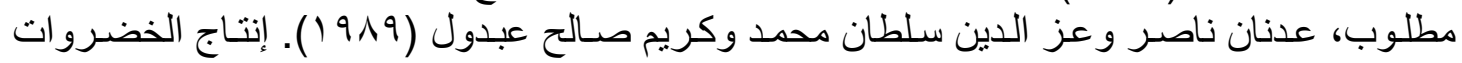

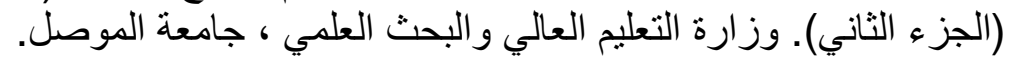

Abdalla, M. H. and A. M. A. Wahab (1995). Response of nitrogen fixation , nodule activities, and growth to potassium supply in water stressed broad bean. J. of Plant Nutrition. 18(7): 1391-1402.

Abdelmula, A.A. and I.K. Abuanja (2007). Genotypic responses, yield stability , and association between characters among some of Sudanese faba bean Vicia faba L. genotypes under Heat stress. Conference on International Agric. Res. for Development. October 9-11.

Alan, O. and. H. Geren (2007). Evaluation of heritability and correlation for seed yield and its components in faba bean Vicia faba L.. J. of Agron., 6(3): 484-487.

Alghamdi, S.S. (2007). Genetic behavior of some selected faba bean genotypes. African. C. Sci. Conference Proceeding. 8.pp. 709-714.

Alghamdi, S.S. and K.A. Ali (2004). Performance of several newly bred faba bean lines. Egypt J. Plant Breed., 8: 189-200.

Allard, R.W. (1960). Principles of Plant Breeding. John Wiley and Sons. Inc. New York.

Anonymous, (2002). Farm Chemicals Hand Book. III Meister Publishing Company. PP.828.

Belitz, H. ; W. Grosch and P. Schieberle (2009). Food Chemistry. $4^{\text {th }}$ ed . Springer. USA.

Cochran, V.L. and S.F. Schlenther (1995). Intercropped oat and faba bean in Alaska-dry matter production, dinitrogen fixation, nitrogen transfer and nitrogen fertilizer response. Agron., H.87(3): 420-424.

Diaz, D. ; M. Morlacchini ; F. Masoero ; M. Moschini ; G. Fusconi and G. Piva (2006). Pea seeds Pisum sativum, faba beans Vicia faba var. minor and lupin seeds Lupinus albus var. multitalia as protein sourcesin broiler diets: effect of extrusionon growth performance. Ital. J. Anim. Sci., 5: 43-53.

Iyad, W. M. ; J. H. Nizar ; M. T. Abdel - Rahman and O. S. Migdadl (2004). The importance of Bee - Pollination in four genotypes of faba bean Vicia faba L.. Int. J. Agri., 6(1): 9-12.

Kahalil, S.A. and W. Erskine (2001). Combatin disease problems of grain legumes in Egypt. Grain Legumes, 32: 24-26.

kalia, P. ; S. Sood and Y. Sing (2003). Genetic variability in faba bean Vicia faba L. for pod yield and its contributing traits. Indian J. Genet., 63(3): 261-262. 
kalia, P. and S. Sood (2004). Genetic variation and association analyses for pod yield and other agronomic and quality characters an Indian Himalayan collection of broad bean Vicia faba L.. Sabrao J. of Breeding and Genetics, 36(2): 55-61.

Karadavut, U. ; C. Palta ; Z. Kavurmacl and Y. Bolek (2010). Some grain yield parameters of multi-environmental trials in faba bean Vicia faba L. genotypes. Int. J. Agric. Biol., 12(2): 217-220.

Mather, K. and J.L. Jinks (1982). Biometrical Genetics. $3^{\text {rd }}$ ed. Chapman and Hall Ltd. London.

Moschini, M. ; F. Masoero ; A. Prandini ; G. Fusconi ; M. Morlacchini and G. Piva (2005). Raw pea Pisum sativum, raw faba beans Vicia faba var. minor and raw lupin Lupinus albus var. multitalia as alternative protein sources in broiler diets. Ital. J. Anim. Sci., 4: 59-69.

Mustafa, A.M.A. (2007). Genetic variation among Egyptian cultivars of Vicia faba L.. Pakistan J. of Biol. Sci., 10(23): 4204-4210.

Nadal, S. ; C. Alfonso ; F. Fernando and T.M. Maria (2005). Effect of growth habit on agronomic characters in faba bean. Agric. Conspectus Scientifics, 70(2): 43-47.

Robinson, H.F. (1966). Quantitative genetics in relation to breeding on the centennial of mendelism. Indian J. Genet., 26 A: 171-187.

Sendecor, G.W. and W. Cochran (1967). Statistical Methods. $6^{\text {th }}$ ed .Iowa State Univ. Press. Ames. USA.

Singh, R.K. and B.D. Chaudhary (1985). Biometrical Methods in Quantitative Genetic Analysis. Kalyani Publishers. New Delhi., India.

Talal, T. and S. Ghalib (2006). Effect of planting date on faba bean Vicia faba L. nodulation and performance under semiarid conditions. World $\mathrm{J}$. of Agric. Sci., 2(4): 477-482.

Toker, C. (2004). Estimates of broad-sense heritability for seed yield and yield criteria in faba bean Vicia faba L.. Hereditas, 140: 222-225.

Ulukan, H. ; M. Guler and S. keskin (2003). A Path coefficient analysis of some yield and yield components in faba bean Vicia faba L. genotypes. Pakistan J. Biol. Sci., 6: 1951-1955.

Walter, A.B. (1975). Manual of Quantitave Genetics [3 rd edition] , Washington State Univ. Press, U.S.A.

Welsh, J.R. (1981). Fundamentals of Plant Genetics and Breeding. John Wiley \&Sons, Inc. New York, USA. 